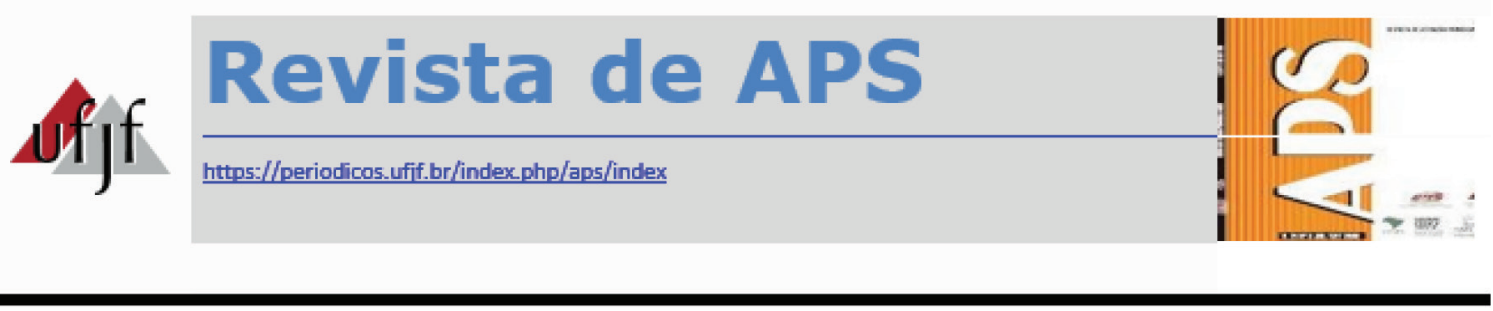

\title{
Oferta de assistência nutricional a crianças menores de cinco anos na Estratégia de Saúde da Família
}

\section{Provision of nutritional assistance to children under the age of five in the Family Health Strategy}

Weslla Karla Albuquerque Silva de Paula ${ }^{1}$, Bianca Santana Silva ${ }^{2}$, Horasa Gonçalves Leal de Oliveira ${ }^{3}$, Priscila Monteiro Tavares de Lavôr ${ }^{4}$, Isabella Chagas Samico ${ }^{5}$

\begin{abstract}
RESUMO
OBJETIVO: Avaliar a oferta da assistência nutricional às crianças menores de cinco anos assistidas pelas equipes da Estratégia Saúde da Família. MÉTODO: Estudo transversal, avaliativo, ancorado no modelo sistêmico de Donabedian, sendo avaliadas as dimensões estrutura e processo. Compuseram a amostra 41 enfermeiros das equipes de Estratégia de Saúde da Família de um município do interior de Pernambuco. RESULTADOS: Os achados do estudo revelam, em relação à estrutura, o item "recursos humanos" como o único classificado na condição de regular, sendo os demais itens pontuados na condição de bons. Quanto ao componente "processo", os itens avaliados apresentaram conformidade, sendo classificados entre as condições de bom e excelente. CONCLUSÃO: Evidenciou-se a necessidade de fomento à formação continuada dos enfermeiros das equipes de Estratégia de Saúde da Família para o desenvolvimento das ações de alimentação e nutrição.
\end{abstract}

PALAVRAS-CHAVE: Deficiências Nutricionais. Avaliação de Serviços de Saúde. Enfermagem. Avaliação nutricional. Estratégia Saúde da Família.

\footnotetext{
${ }^{1}$ Doutora em Saúde Materno-infantil. Professora Assistente do Curso de Bacharelado em Enfermagem da Associação Caruaruense de Ensino Superior e Técnico. Faculdade ASCES. Caruaru, PE, Brasil. E-mail: weslla19@hotmail.com

${ }^{2}$ Bacharela em Enfermagem. Associação Caruaruense de Ensino Superior e Técnico. Faculdade ASCES. Caruaru, PE, Brasil.

${ }^{3}$ Bacharela em Enfermagem. Associação Caruaruense de Ensino Superior e Técnico. Faculdade ASCES. Caruaru, PE, Brasil.

${ }^{4}$ Bacharela em Enfermagem. Associação Caruaruense de Ensino Superior e Técnico. Faculdade ASCES. Caruaru, PE, Brasil.

${ }^{5}$ Doutora. Docente da Pós-graduação em Saúde Materno-Infantil. Instituto de Medicina Integral Prof. Fernando Figueira (IMIP). Recife, PE, Brasil.
} 


\begin{abstract}
OBJECTIVE: To evaluate the provision of nutritional assistance to children under the age of five assisted by the Family Health Strategy teams. METHOD: Cross-sectional, evaluative study, anchored in the Donabedian systemic model that evaluated the size structure and the process. The sample comprised 41 nurses from the Family Health Strategy teams in a municipal district in Pernambuco. RESULTS: The findings of the study reveal, in relation to the structure, the item "human resources" as the only one classified in the condition of regular, while the other items were scored as good. As for the "process" component, the evaluated items presented conformity, being classified between good and excellent conditions. CONCLUSION: It was evidenced that it is necessary to foster the continuous formation of the nurses from the teams of Strategy of Health of the Family for the development of the feeding and nutrition actions.
\end{abstract}

KEYWORDS: Deficiency Diseases. Health Services Evaluation. Nursing. Nutrition Assessment. Family Health Strategy.

\title{
INTRODUÇÃO
}

Nas últimas décadas têm-se observado mudanças no padrão alimentar e de saúde da população brasileira. E essas mudanças desenham um novo cenário de problemas de saúde relacionados à alimentação e nutrição, sobretudo o infantil'1.

As deficiências nutricionais correlacionam-se aos déficits no crescimento e desenvolvimento das crianças, advindas da ingestão inadequada dos alimentos, deixando-as suscetíveis às doenças carenciais específicas, a exemplo da anemia ferropriva e deficiência de vitamina A, e ao mesmo tempo ao desenvolvimento precoce de sobrepeso e obesidade. Essas situações podem resultar agravos à saúde, como alterações no sistema imunológico, cognitivo, cardiovascular, além do aumento da morbimortalidade infantil ${ }^{1-3}$.

Diante de tais implicações, torna-se indispensável a inclusão do estado nutricional infantil na agenda das prioridades de saúde coletiva, atentando a necessidade de essa demanda ser trabalhada na atenção primária. A Estratégia Saúde da Família (ESF) entendida como um modelo de reorientação da atenção básica desenvolve ações de promoção à saúde, prevenção e recuperação de agravos. Especificamente, na atenção à saúde da criança, a ESF executa atividades de incentivo ao aleitamento materno e práticas alimentares saudáveis, imunização, atenção às doenças prevalentes na infância, como também acompanha o crescimento e desenvolvimento infantil, participa no combate às carências nutricionais, entre outras ações ${ }^{4}$.

Com base na importância dessas práticas, estudos avaliativos são necessários. A institucionalização da avaliação na atenção básica, com a inserção do monitoramento, atravésdaconstruçãode processosestruturadosesistemáticos, serveparaqualificarasações e garantir a qualidade da assistência prestada pelos serviços de saúde $\mathrm{e}^{5-6}$. Os estudos nessa 
área utilizam-se de parâmetros que servem como fundamento para avaliação da qualidade de saúde e muitas dessas investigações têm sevalido do modelosistêmico de Donabedian, o qual propõe a tríade estrutura, processo e resultado ${ }^{5}$.

Nessa perspectiva, foram levantados questionamentos sobre a adequação das condições das equipes da ESF para desenvolverem as ações programáticas de alimentação e nutrição na atenção primária. Diante da escassez de estudos sobre a avaliação da oferta nutricional na população infantil assistida pela ESF e com esse tipo de abordagem metodológica, objetivou-se avaliar a oferta da assistência nutricional às crianças menores de cinco anos assistidas pelas equipes da Estratégia Saúde da Família em um município do interior de Pernambuco.

\section{MÉTODO}

Trata-se de um estudo transversal de natureza quantitativa, realizado em unidades de Estratégia de Saúde da Família de um município do interior de Pernambuco. O município está situado na região agreste do estado de Pernambuco, faz parte da IV Gerência Regional de Saúde (IV GERES) e possui população estimada de 356.872 habitantes, segundo estimativas do ano de 2018 do Instituto Brasileiro de Geografia e Estatística (IBGE), distribuída em 920,611 km².

Consideraram-se como critério de inclusão profissionais médicos e/ou enfermeiros das unidades de saúde da família, cujas equipes estivessem trabalhando em conjunto há pelo menos seis meses. A escolha por esses profissionais se deu porque eles compõem a equipe básica da ESF e prestam atenção nutricional à criança, seja por meio de ações individuais ou coletivas. Já o nutricionista, considerado especialista nessa temática, faz parte da equipe de apoio ao Saúde da Família e, como um dos propósitos era identificar a existência do sistema de referência e contrarreferência na oferta da assistência nutricional, optou-se por não incluí-lo.

Para fins de cálculo de amostra foi utilizado o software estatístico Epi Info 7, a partir das seguintes informações disponíveis na época da elaboração do projeto de pesquisa: população total de equipes de saúde da família implantadas no município (total de 43) uma frequência de 50\%, um erro estimado de $3 \%$ e um nível de confiança (IC) de $95 \%$, obtendo-se a amostra total de 41 equipes de saúde da família. A amostragem para escolha das equipes foi por conveniência.

Tomou-se como referência para a avaliação de conformidade da estrutura e processo das unidades de saúde da família a Portaria 2488/11, que, à época da realização da pesquisa, era a Política Nacional de Atenção Básica ${ }^{4}$ vigente, a Política Nacional de Alimentação e Nutrição ${ }^{7}$ e a Política Nacional de Atenção Integral à Saúde da Criança: orientações para implementação ${ }^{8}$. Ainda, foram utilizadas como parâmetros as normas do Caderno de Atenção Básica: Carências de Micronutrientes ${ }^{9}$, Caderno de Atenção 
Básica no 23 Saúde da Criança: Aleitamento materno e alimentação complementar ${ }^{10}$, Caderno de Atenção Básica no 27 sobre diretrizes do NASF: Núcleo de Apoio a Saúde da Família $^{11}$ e manual de estrutura física das unidades básicas de saúde ${ }^{12}$.

A estrutura diz respeito às particularidades dos provedores, instrumentos, recursos e características físicas e organizacionais dos serviços ${ }^{13}$. A avaliação do componente estrutura considerou os itens: instalação física, recursos materiais, recursos humanos, normas e rotinas.

O item instalação física foi avaliado através de observação direta das salas de espera, de atividades educativas, consultório multiprofissional e local para dispensação dos medicamentos.

Com relação ao item recursos materiais, consistiu-se em verificar, através da observação direta e entrevistas com os profissionais, a disponibilidade de sulfato ferroso em gotas e da vitamina A (100.000 e $200.000 \mathrm{UI}$ ), assim como de material educativo sobre alimentação saudável para realização das atividades educativas, presença de antropômetro, balança eletrônica ou mecânica, fita métrica e a presença da caderneta de saúde da criança do Ministério da Saúde.

O item recursos humanos levou em consideração a normatização da Portaria 2488/114. Esses dados foram obtidos através de entrevista com profissionais das unidades de saúde da família, quanto às variáveis especialização na área e participação dos profissionais - médico e enfermeiro - em programas de capacitação desenvolvidos pela Secretaria de Saúde e a observação das fichas SSA2 e PMA2 do sistema de informação da atenção básica (SIAB) referentes ao mês anterior à coleta dos dados. Também foi avaliada a presença da equipe de saúde mínima (1 médico, 1 enfermeiro, 1 auxiliar ou técnico de enfermagem, 1 agente comunitário de saúde (ACS) para no máximo 750 habitantes cobertos e uma equipe de saúde da família para no máximo 4000 habitantes cobertos).

Quanto aos aspectos sobre normas e rotinas, verificou-se a presença de rotinas técnicas de procedimento escrito sobre acompanhamento do crescimento e desenvolvimento da criança, as orientações quanto alimentação, utilização da caderneta de saúde da criança nas consultas de puericultura, solicitação de exames bioquímicos, registro de manutenção preventiva das balanças e registro das atividades educativas em grupo desenvolvidas pelas equipes da ESF.

O processo corresponde às atividades, bens e serviços prestados e a sua adequação ${ }^{13}$. No que concerne à avaliação desse componente, foram analisados os itens: avaliação clínica, análise da caderneta de saúde da criança, promoção de atividades educativas e sistema de referência e contrarreferência.

O item avaliação clínica contemplou a realização do acompanhamento do crescimento e desenvolvimento infantil (exame físico e aferição de medidas antropométricas). Na análise da caderneta de saúde da criança (CSC), foi observado o 
preenchimento pelos profissionais de saúde nas consultas de puericultura, com base nas orientações do manual para utilização da CSC.

No item promoção de atividades educativas, foi verificada a realização dessas atividades em grupo ou individual, através de informações obtidas por meio de entrevista com profissionais de saúde da família e registro dessas atividades.

Por fim, no item sistema de referência e contrarreferência, foi indagado o encaminhamento para outros profissionais (equipes do Núcleo de Apoio à Saúde da Família - NASF), utilização de ficha de referência e existência de contrarreferência e laboratório de referência para realização de exames.

A coleta dos dados aconteceu no período de junho a agosto de 2014, sendo realizada por três das pesquisadoras. Para tal, utilizou-se um questionário estruturado. Cada item dos componentes estrutura e processo foi consolidado e classificado de acordo com o grau de conformidade a partir das normas: excelente (90 a 100\%), bom (70 a 89\%), regular (50 a 69\%) e ruim (<50\%). Foi realizada análise descritiva, com apresentação das frequências simples e absoluta das variáveis investigadas.

O presente estudo foi aprovado pelo Comitê de Ética em Pesquisa do Centro Universitário Tabosa de Almeida (ASCES-UNITA) da Associação Caruaruense de Ensino Superior, sob protocolo número 532.406 (no CAAE: 22391313.2.0000.5203), atendendo às normas regulamentares de pesquisas que envolvem seres humanos, conforme Resolução 466/2012 do Conselho Nacional de Saúde.

\section{RESULTADOS}

Os resultados estão apresentados de acordo com a análise descritiva dos elementos estrutura e processo, na oferta da assistência nutricional das crianças menores de cinco anos das Estratégias de Saúde da Família elencadas para o estudo. Em 100\% das unidades amostradas, o profissional enfermeiro foi o respondente do questionário. Na tabela 1, estão descritas as características do componente estrutura em relação à instalação física, recursos materiais, recursos humanos e normas e rotinas das equipes de saúde da família.

Das 41 unidades, quatro $(9,76 \%)$ ainda não possuíam consultório médico e de enfermagem de acordo com as necessidades para desenvolver as ações de sua competência. Quanto à existência de área/sala apropriada para realização de atividades educativas, apenas vinte e sete $(65,85 \%)$ dispunham desse espaço. Em doze unidades $(29,27 \%)$ ainda não havia um local para arquivos e registros e duas $(4,88 \%)$ não possuíam sala de vacina.

No que se refere aos recursos materiais, perceberam-se alguns pontos de fragilidade. Apesar de trinta e sete $(90,24 \%)$ das unidades de saúde disporem de sulfato ferroso e trinta e oito $(92,68 \%)$ da megadose de vitamina $A$, observou-se que vinte e 
quatro unidades $(58,54 \%)$ não apresentavam quantidade de medicamentos suficiente para atender a demanda mensal da unidade.

Quando indagado aos enfermeiros com que intensidade os medicamentos faltaram nos últimos seis meses, um terço dos entrevistados $(33,3 \%)$ respondeu que os mesmos acabam logo no início do período, ficando a maior parte do mês sem medicamentos. Ressalta-se, também, que treze unidades $(31,71 \%)$ ainda não tinham material educativo sobre alimentação saudável para realização de palestras.

Com relação aos recursos humanos, dezessete $(41,46 \%)$ profissionais das unidades de saúde da família afirmaram não apresentar capacitação para executar as ações de alimentação e nutrição na atenção primária, previstas pelo Ministério da Saúde. Ademais, sete equipes não apresentaram conformidade quanto à presença da equipe mínima. Apenas nove (21,9\%) ESF possuíam menos de 1.000 famílias cadastradas (média de 1.480 famílias/Unidades de Saúde da Família), com número de famílias variando entre 435 até 2.800 . Verificou-se que quinze (36,59\%) unidades tinham número de agentes comunitários de saúde insuficiente para cobrir $100 \%$ da população cadastrada.

Quanto às normas e rotinas, duas unidades não tinham acesso ao manual de acompanhamento do crescimento e desenvolvimento da criança. Dez (24,39\%) unidades não possuíam o caderno de atenção básica no 23 e treze (31,71\%) não faziam registros da manutenção preventiva das balanças eletrônicas ou mecânicas. O único item em conformidade foi quanto à solicitação de exames bioquímicos, todas as unidades faziam.

Tabela 1 - Descrição e frequência dos itens avaliados nas Unidades de Saúde da Família referentes ao componente estrutura. Caruaru-PE, 2014

\begin{tabular}{lc}
\hline Instalações físicas & Sim \\
N (\%) \\
\hline Unidade de Saúde da Família com consultório médico e de enfermagem, de acordo & 37 \\
com as necessidades de desenvolvimento do conjunto de ações de sua competência & $(90,24)$ \\
Unidade de Saúde da Família com área/sala para realização de atividades educativas & 27 \\
Unidade de Saúde da Família com área/sala de espera para clientes que aguardam & 40 \\
o atendimento & $(97,56)$ \\
Unidade de Saúde da Família com local para dispensação dos medicamentos & 39 \\
Unidade de Saúde da Família com local para arquivos e registros & $(95,12)$ \\
& $(70,73)$ \\
Unidade de Saúde da Família com sala de cuidados básicos de enfermagem & $(87,80)$ \\
& 39 \\
Unidade de Saúde da Família com sala de vacina & $(95,12)$ \\
& 37 \\
Unidade de Saúde da Família com disposição do xarope de sulfato ferroso & $(90,24)$ \\
Unidade de Saúde da Família com disposição das megadoses de vitamina A & 38 \\
(100.000 e 200.000 UI) & $(92,68)$ \\
Quantidade de medicamentos disponibilizada no período atende a demanda da & 17 \\
USF & $(41,46)$ \\
\hline
\end{tabular}


(Conclusão)

\begin{tabular}{lc}
\hline Instalações físicas & Sim \\
& N (\%) \\
\hline A unidade de saúde dispõe de material educativo sobre alimentação saudável para & 28 \\
realização de palestras? & $(68,29)$ \\
Balança eletrônica ou mecânica & 41 \\
& $(100)$ \\
Fita métrica & 41 \\
Antropômetro & $(100)$ \\
Caderneta de saúde da criança & $(87,80)$ \\
Equipe de Saúde da Família capacitada para executar as ações de alimentação e & 36 \\
nutrição na atenção primária previstas pelo Ministério da Saúde & 24 \\
Profissionais médicos e/ou enfermeiro que realizaram o curso introdutório em & $(58,54)$ \\
saúde da família* & \\
Enfermeiro & 28 \\
Profissionais médicos e/ou enfermeiro que fizeram curso de especialização em & $(68,29)$ \\
saúde da Família* & \\
Enfermeiro & 28 \\
& $(68,29)$ \\
Equipe de Saúde da Família em conformidade quanto à presença da equipe mínima\# & 34 \\
Equipe de Saúde da Família com número de Agentes Comunitários de Saúde (ACS) & $(82,93)$ \\
suficiente para cobrir 100\% (cem por cento) da população cadastrada, com um & 26 \\
máximo de 750 pessoas por ACS e de até 12 (doze) ACS por ESF. & $(63,41)$ \\
Acesso ao manual de acompanhamento do crescimento e desenvolvimento da & 39 \\
criança na unidade & $(95,12)$ \\
Acesso ao Caderno de Atenção Básica no 23 sobre saúde da criança: nutrição & 31 \\
infantil na unidade & $(75,61)$ \\
Utilização da caderneta de saúde da criança nas condutas de puericultura & 40 \\
Solicitação de exames bioquímicos & $(97,56)$ \\
Registro da manutenção preventiva das balanças & 41 \\
Registro das atividades educativas em grupo desenvolvidas pela equipe de Saúde & $(100)$ \\
da Família & 28 \\
\hline & $(68,29)$ \\
& 37 \\
& $(90,24)$ \\
\hline
\end{tabular}

*Informação disponível apenas para o profissional enfermeiro. \# Equipe mínima: 1 médico, 1 enfermeiro, 1 auxiliar ou técnico de enfermagem, 1 agente comunitário de saúde (ACS) para no máximo 750 habitantes cobertos e uma equipe de saúde da família para no máximo 4.000 habitantes cobertos

Fonte: elaborada pelas autoras

A tabela 2 apresenta as informações obtidas quanto ao componente processo. Em todas as unidades de saúde, os profissionais afirmaram realizar avaliação clínica (exame físico e aferição de medidas antropométricas), acompanhando o crescimento e desenvolvimento das crianças menores de cinco anos. 
Quanto à promoção de atividades educativas sobre alimentação e nutrição, apesar de trinta e oito $(92,68 \%)$ enfermeiros afirmarem realizá-las, treze $(31,71 \%)$ não registravam as informações nos prontuários.

No que concerne ao item sistema de referência, trinta e oito $(92,68 \%)$ unidades encaminhavam as crianças de risco nutricional para níveis de maior complexidade, enquanto oito $(19,51 \%)$ unidades de saúde não faziam encaminhamento de crianças em risco nutricional para profissionais do Núcleo de Apoio à Saúde da Família (NASF).

Ainda referente ao sistema de referência e contrarreferência, em dezenove unidades $(46,34 \%)$ não era realizado encaminhamento formalizado para a referência (utilização de ficha de referência e contrarreferência) com garantia de atendimento. Vinte dos profissionais das ESF afirmaram ter dificuldades para marcar a consulta de crianças em risco nutricional para o profissional de referência. Aproximadamente $93 \%$ das unidades encaminhavam as crianças ao laboratório de referência para realização de exames, porém pouco mais de $68 \%$ relataram atraso no recebimento dos resultados.

Tabela 2 - Descrição e frequência dos itens avaliados nas Unidades de Saúde da Família referentes ao componente processo. Caruaru-PE, 2014

\begin{tabular}{|c|c|}
\hline Avaliação clínica & Sim \\
\hline $\begin{array}{l}\text { Acompanhamento do crescimento e desenvolvimento infantil (exame físico e } \\
\text { aferição de medidas antropométricas) }\end{array}$ & $\begin{array}{c}41 \\
(100)\end{array}$ \\
\hline$<1$ ano & $\begin{array}{c}41 \\
(100)\end{array}$ \\
\hline \multicolumn{2}{|l|}{ Mensal } \\
\hline$<2$ anos & $\begin{array}{c}41 \\
(100)\end{array}$ \\
\hline \multicolumn{2}{|l|}{ Mensal } \\
\hline \multicolumn{2}{|l|}{ Bimestral } \\
\hline \multicolumn{2}{|l|}{ Trimestral } \\
\hline \multicolumn{2}{|l|}{ Semestral } \\
\hline \multicolumn{2}{|l|}{ Anual } \\
\hline$<5$ anos & $\begin{array}{c}41 \\
(100)\end{array}$ \\
\hline \multicolumn{2}{|l|}{ Mensal } \\
\hline \multicolumn{2}{|l|}{ Bimestral } \\
\hline \multicolumn{2}{|l|}{ Trimestral } \\
\hline \multicolumn{2}{|l|}{ Semestral } \\
\hline \multicolumn{2}{|l|}{ Anual } \\
\hline Registro dessas informações no prontuário & $\begin{array}{c}41 \\
(100)\end{array}$ \\
\hline $\begin{array}{l}\text { Preenchimento da caderneta de saúde da criança durante as consultas de } \\
\text { puericultura }\end{array}$ & $\begin{array}{c}40 \\
(97,56)\end{array}$ \\
\hline $\begin{array}{l}\text { Promoção de atividades educativas, em grupo ou individuais, sobre alimentação } \\
\text { e nutrição }\end{array}$ & $\begin{array}{c}38 \\
(92,68)\end{array}$ \\
\hline
\end{tabular}


(Conclusão)

\begin{tabular}{lc}
\hline Avaliação clínica & Sim \\
\hline Registro dessas informações no prontuário & $28)$ \\
Sistema de referência e contrarreferência & $(68,29)$
\end{tabular}

Encaminhamento de crianças em risco nutricional para outros níveis de atenção à saúde (maior complexidade)

Encaminhamento de crianças em risco nutricional para profissionais das equipes de apoio (Núcleo de Apoio à Saúde da Família - NASF)

Encaminhamento para a referência formalizado (utilização de ficha de referência e contrarreferência) com garantia de atendimento

Dificuldade para marcar a consulta de crianças em risco nutricional para o profissional de referência

Laboratório de referência para realização de exames

Tempo de retorno do resultado do exame adequado

Fonte: elaborado pelas autoras

A tabela 3 sumariza as informações segundo o grau de conformidade dos componentes avaliados. Quanto à estrutura, apenas a dimensão recursos humanos foi classificada como regular. No que concerne ao componente processo, as dimensões foram classificadas como bom e excelente.

Tabela 3 - Classificação das Unidades de Saúde da Família segundo grau de conformidade aos componentes estrutura e processo. Caruaru-PE, 2014

\begin{tabular}{lcc}
\hline \multicolumn{1}{c}{ Componentes } & Conformidade & Classificação \\
\hline Estrutura & & \\
Instalação física & $86 \%$ & Bom \\
Recursos materiais & $84 \%$ & Bom \\
Recursos humanos & $67 \%$ & Regular \\
Normas e rotinas & $88 \%$ & Bom \\
Processo & & \\
Avaliação clínica & $100 \%$ & Excelente \\
Análise da CSC & $98 \%$ & Excelente \\
Promoção de atividades educativas & $80 \%$ & Bom \\
Sistema de referência e contrarreferência & $73 \%$ & Bom \\
\hline * CSC - Caderneta de Saúde da Criança; Classificação: excelente (90 a 100\%), bom (70 a 89\%), regular (50 \\
a 69\%) e ruim (< 50\%)
\end{tabular}

Fonte: elaborado pelas autoras 


\section{DISCUSSÃO}

O presente estudo permitiu a avaliação da estrutura e processo das unidades de saúde da família em um município no interior pernambucano. O fato de os enfermeiros terem sido os únicos respondentes do questionário da pesquisa se deu por apresentarem maior disponibilidade no momento da coleta dos dados, apesar de os critérios de elegibilidade não privilegiarem apenas esse profissional. O enfermeiro tem sido reconhecido como um importante membro da equipe multidisciplinar, na rede primária de assistência à saúde, pois se apresenta como um dos principais mediadores da equipe de saúde e da comunidade assistida. Cabem a ele atividades de coordenação, supervisão, treinamento, planejamento de ações educativas, controle da equipe, entre outras especificidades ${ }^{4,14}$.

No que concerne ao componente estrutura, no item instalações físicas, observouse que a maioria das unidades estava em conformidade com o preconizado. Entretanto, verificou-se que ainda persistem algumas limitações. Em um estudo realizado para avaliar o desempenho da atenção primária à saúde no município de Belo Horizonte-MG, identificou-se que a boa estrutura física promove melhores práticas dos profissionais de saúde, favorecendo a qualidade técnica requerida, conforto e condições humanizadas de trabalho para a equipe ${ }^{15}$.

Dentre essas barreiras estruturais, percebeu-se a ausência de um local específico para a realização das atividades educativas e, por vezes, estas eram realizadas na sala de espera. Assemelha-se ao resultado encontrado em um estudo realizado no município Campina Grande-PB o qual mostrou que muitas das dificuldades para realização das ações educativas advinham da inadequação das instalações físicas ${ }^{16}$. Quando não há espaço próprio para realização de tais ações, ocorre a dispersão do público-alvo, prejudicando o entendimento e a discussão sobre o assunto enfatizado.

No que se refere à disposição de medicamentos, a maioria das Unidades Básicas de Saúde (UBS) dispunham de sulfato ferroso e megadoses de vitamina A, porém os medicamentos eram insuficientes para atender a demanda mensal. Estudo que caracterizou a disponibilidade de medicamentos em UBS no Brasil observou maior disponibilidade de antianêmicos, vitamínicos e sais minerais em municípios de até 500 mil habitantes, em UBS com ESF, especialmente naquelas cujas equipes aderiram ao Programa de Melhoria do Acesso e da Qualidade da Atenção Básica (PMAQ-AB) ${ }^{17}$. A disposição desses suplementos alimentares pode conferir menor carência nutricional em crianças privadas de alimentação diversificada e rica nesses minerais.

Ainda em relação ao item recursos materiais, verificou-se que em algumas unidades não havia materiais para execução de atividades educativas, bem como para avaliação nutricional das crianças. Esse resultado contraria o observado no estudo em unidades de atenção primária à saúde do município de Bauru-SP, o qual evidenciou que 
todas as ESF possuíam os instrumentos para verificação das medidas antropométricas ${ }^{18}$. A lacuna de equipamentos adequados no momento da realização de ações educativas dificulta ou limita a compreensão da comunidade. Do mesmo modo, a ausência do antropômetro pode induzir os profissionais a realizarem uma avaliação nutricional por meio do improviso, ou até mesmo a não realização, podendo exibir falhas no acompanhamento do crescimento infantil.

No que diz respeito aos recursos humanos, notou-se limitações quanto à capacitação dos profissionais frente às ações de alimentação e nutrição infantil. A capacitação teórico-prática e a educação continuada das equipes de saúde da família são fundamentais para a plena inserção de todos os profissionais no cuidado com a criança ${ }^{19}$. A ausência de cursos de capacitação e treinamento profissional origina dificuldades na prestação de serviço àqueles que devem ser atendidos, podendo incorrer na adoção de condutas desarticuladas das evidências científicas vigentes.

Ainda na avaliação desse componente, percebeu-se que a maioria das equipes da ESF trabalhava com número excessivo de famílias cadastradas, em desacordo com a $\mathrm{PNAB}^{4}$ vigente à época. Essa inconformidade pode predispor a dificuldades na realização das ações voltadas à saúde da criança, particularmente aquelas que dizem respeito à promoção da saúde e prevenção de agravos.

No item normas e rotinas, apesar de a maioria dos profissionais das unidades ter acesso aos manuais de acompanhamento e orientação nutricionais e a exames laboratoriais, muitos não realizavam o registro da manutenção das balanças utilizadas, corroborando com o achado de outro estudo ${ }^{20}$. Tal medida é importante uma vez que aferição incorreta do peso implica a avaliação nutricional errônea e, consequentemente, a adoção de condutas inadequadas.

Quanto ao componente processo, a frequência na execução do acompanhamento do crescimento e desenvolvimento mostrou-se em conformidade com as orientações do Ministério da Saúde (MS), o que contraria os dados encontrados em uma pesquisa efetuada na unidade de saúde de um município do centro-sul paranaense, onde a consulta de puericultura se limitava à avaliação dos agravos instalados na criança ${ }^{21}$. Um calendário mínimo de consultas de puericultura permite acompanhar o progresso do infante, permitindo identificar aqueles em maior risco de morbimortalidade, além de sinalizar o alarme precoce para os desvios nutricionais, propiciando o enfoque nas ações de promoção e prevenção e não naquelas meramente curativas.

Quanto ao item promoção de atividades educativas, estas eram realizadas, contudo nem sempre registradas. Os registros dos cuidados são fundamentais para que os profissionais de saúde estejam legitimados de todas as ações que foram realizadas com o usuário e sua família ${ }^{21}$. A ausência de registro traz consequências no rendimento, retrabalho, dificuldade no atendimento das crianças e na continuidade dos cuidados prestados. 
No item sistema de referência e contrarreferência, foram evidenciadas barreiras organizacionais nas unidades de saúde, a exemplo da dificuldade de acesso aos serviços especializados, bem como no retorno dos exames laboratoriais em tempo oportuno. Esse resultado corrobora com outro estudo, no qual as falhas no processo de comunicação para referência e contrarreferência entre os profissionais também foram observados ${ }^{22}$. Enfatizase que a dificuldade no acesso interfere na coordenação do cuidado ao usuário na Rede de Atenção à Saúde (RAS), importante indicador de qualidade dos serviços de Atenção Básica.

Os resultados aqui apresentados não permitiram analisar a tríade que compõe a avaliação da qualidade dos serviços, visto que apenas os componentes estrutura e processo foram avaliados. O estudo traz limitações próprias do referencial adotado e do fato de apenas os profissionais enfermeiros terem participado das entrevistas, não sendo possível avaliar a oferta nutricional às crianças sob a ótica dos médicos das equipes, que também assistem esse grupo etário. Apesar de a amostragem para seleção das unidades ter sido realizada por conveniência, o que se configuraria como uma limitação, apenas duas unidades do município não foram analisadas, o que não invalida os resultados observados, bem como a utilização dos mesmos.

\section{CONCLUSÃO}

A despeito da inconformidade de alguns critérios que integram os componentes estrutura e processo, apenas o item recursos humanos foi classificado como regular, o que sinaliza a necessidade de aperfeiçoamento e capacitação das equipes de saúde da família, no que se reporta às ações de alimentação e nutrição e à formação continuada desses profissionais.

Por se tratar de um estudo transversal, este pode servir de linha de base para estudos subsequentes sobre o tema, assim como colaborar para que profissionais e gestores reflitam suas práticas, auxiliando-os nas tomadas de decisão frente às necessidades encontradas.

\section{REFERÊNCIAS}

1. França AKTC, Chagas, DC, Pessoa, FS, Pinho, JRO, Cabral, NAL. Alimentação e nutrição na atenção básica em saúde. 1. ed. São Luiz: EDUFMA; 2017.

2. Carvalho CA, Fonsêca PC, Priore SE, Franceschini SCC, Novaes JF. Food consumption and nutritional adequacy in Brazilian children: a systematic review. Rev Paul Pediatr [Internet]. 2015 [citado em 2019 mar 08]; 33(2):211-21. Disponível em: http://www. ncbi.nlm.nih.gov/pmc/articles/PMC4516376/pdf/0103-0582-rpp-33-02-00211.pdf.

3. Romanã DL, Olivares M, Brito A. Prevalence of Micronutrient Deficiencies in Latin America and the Caribbean. Food and Nutrition Bulletin [Internet]. 2015 [citado em 
2019 mar 08]; 36(2):95-97. Disponível em: http://fnb.sagepub.com/content/36/2_ suppl/S95.full.pdf+html.

4. Brasil. Ministério da Saúde. Secretaria de Atenção à Saúde. Departamento de Atenção Básica. Política Nacional de Atenção Básica [Internet]. Brasília; 2012 [citado em 2015 ago 10]. Disponível em: http://189.28.128.100/dab/docs/publicacoes/ geral/pnab.pdf.

5. Facchini LA, Tomasi E, Dilélio AS. Qualidade da Atenção Primária à Saúde no Brasil: avanços, desafios e perspectivas. Saúde Debate [Internet]. 2018 [citado em 2019 mar 8];42(1):208-223. Disponível em: http://www.scielo.br/pdf/sdeb/ v42nspe1/0103-1104-sdeb-42-spe01-0208.pdf.

6. Sousa AN. Monitoramento e avaliação na atenção básica no Brasil: a experiência recente e desafios para a sua consolidação. Saúde Debate [Internet]. 2018 [citado em 2019 mar 8]; 42(1):289-301. Disponível em: http://www.scielo.br/pdf/sdeb/ v42nspe1/0103-1104-sdeb-42-spe01-0289.pdf.

7. Brasil. Ministério da Saúde. Secretaria de Atenção à Saúde. Departamento de Atenção Básica. Política Nacional de Alimentação e Nutrição [Internet]. Brasília; 2013 [citado em 2015 set 20]. Disponível em: http://bvsms.saude.gov.br/bvs/ publicacoes/politica_nacional_alimentacao_nutricao.pdf.

8. Brasil. Ministério da Saúde. Secretaria de Atenção à Saúde. Departamento de Ações Programáticas Estratégicas. Política Nacional de Atenção Integral à Saúde da Criança: orientações para implementação [Internet]. Brasília; 2018 [citado em 2019 mar 8]. Disponível em: http://www.saude.pr.gov.br/arquivos/File/Politica_Nacional_de_ Atencao_Integral_a_Saude_da_Crianca_PNAISC.pdf.

9. Brasil. Ministério da Saúde. Unicef. Cadernos de Atenção Básica: Carências de Micronutrientes [Internet]. Brasília; 2007 [citado em 2019 mar 8]. Disponível em: http://bvsms.saude.gov.br/bvs/publicacoes/cadernos_atencao_basica_carencias_ micronutrientes.pdf.

10. Brasil. Ministério da Saúde. Secretaria de Atenção à Saúde. Departamento de Atenção Básica. Cadernos de Atenção Básica n. 23. Saúde da criança: aleitamento materno e alimentação complementar [Internet]. Brasília; 2015 [citado em 2019 mar 8]. Disponível em: http://bvsms.saude.gov.br/bvs/publicacoes/saude_crianca_ aleitamento_materno_cab23.pdf.

11. Brasil. Ministério da Saúde. Secretaria de Atenção à Saúde. Departamento de Atenção Básica. Cadernos de Atenção Básica n. 39. Núcleo de apoio à saúde da família - Volume 1. Ferramentas para a gestão e para o trabalho cotidiano [Internet]. Brasília; 2014 [citado em 2019 mar 8]. Disponível em: http://bvsms. saude.gov.br/bvs/publicacoes/nucleo_apoio_saude_familia_cab39.pdf.

12. Brasil. Ministério da Saúde. Departamento de Atenção Básica. Manual de estruturafísica das unidades básicas de saúde: saúde da família [Internet]. Brasília; 
2008 [citado em 2019 mar 8]. Disponível em: http://bvsms.saude.gov.br/bvs/ publicacoes/manual_estrutura_fisica_ubs.pdf.

13. Araujo JP, Viera CS, Oliveira BRG, Gaiva MA, Rodrigues RM. Avaliação dos atributos essenciais da Atenção Primária à Saúde da criança. Rev Bras Enferm [Internet]. 2018 [citado em 2019 mar 8]; 71(Suppl 3):1366-72.

14. Ramos CFV. Práticas educativas: pesquisa-ação com enfermeiros da Estratégia de Saúde da Família. Rev Bras Enferm [Internet]. 2018 [citado em 2019 mar 8]; 71(3):1211-8. Disponível em: http://www.scielo.br/pdf/reben/v71n3/pt_0034-7167reben-71-03-1144.pdf.

15. Turci MA, Costa MFL, Macinko J. Influências de fatores estruturais e organizacionais no desempenho da atenção primária à saúde em Belo Horizonte, Minas Gerais, Brasil, na avaliação de gestores e enfermeiros. Cad Saúde Pública [Internet]. 2015 [citado em 2019 mar 8]; 31(9):1941-52. Disponível em: http://www.scielo.br/pdf/ csp/v31n9/0102-311X-csp-31-9-1941.pdf.

16. Pedraza DF, Menezes TN, Costa GMC. Ações de alimentação e nutrição na estratégia saúde da família: estrutura e processo de trabalho. Rev Enferm UERJ [Internet]. 2016 [citado em 2019 mar 8]; 24(4). Disponível em: http://www.facenf.uerj.br/ v24n4/v24n4a11.pdf.

17. Mendes LV, Campos MR, Chaves GC, Silva RM, Freitas PS, Costa KS, et al. Disponibilidade de medicamentos nas unidades básicas de saúde e fatores relacionados: uma abordagem transversal. Rev SauDeb [Internet]. 2014 [citado em 2015 set 10]; 38:109-23. Disponível em: http://www.scielo.br/pdf/sdeb/ v38nspe/0103-1104-sdeb-38-spe-0109.pdf.

18. Ferreira MCS, Negri F, Galesi LF, Detregiachi CRP, Oliveira MRM. Monitoramento nutricional em unidades de atenção primária à saúde. RASBRAN - Revista da Associação Brasileira de Nutrição [Internet]. 2017 [citado em 2019 mar 8]; 8(1): 37-45. Disponível em: https://www.rasbran.com. $\mathrm{br} / \mathrm{rasbran} /$ article/view/227.

19. Vieira DS et al. A prática do enfermeiro na consulta de puericultura na estratégia saúde da família. Texto \& Contexto - Enfermagem [Internet]. 2018 [citado em 2019 mar 8]; 27(4).Disponível em: http://www.scielo.br/scielo. php?script=sci_arttext\&pid=S0104-07072018000400318\&Ing=pt\&tlng=pt.

20. Niquini RP, Bittencourt AS, Lacerda EMA, Saunders C, Leal MC. Avaliação da estrutura de sete unidades de saúde da família para a oferta da assistência nutricional no pré-natal no município do Rio de Janeiro, Brasil. Rev Bras Saude MaterInfant [Internet]. 2010 [citado em 2015 set 10]; 10(1):61-8. Disponível em: http://www.scielo.br/pdf/rbsmi/v10s1/06.pdf. 
21. Baratieri T, Soares LG, Botti ML, Campanini AC. Consulta de enfermagem em puericultura: um enfoque nos registros de atendimentos. Rev Enferm UFSM [Internet]. 2014 [citado 2019 mar 8]; 4(1):206-16. Disponível em: https://periodicos. ufsm.br/reufsm/article/view/8553.

22. Protasio APL et al Avaliação do sistema de referência e contrarreferência do estado da Paraíba segundo os profissionais da Atenção Básica no contexto do 10 ciclo de Avaliação Externa do PMAQ-AB. Saúde Debate [Internet]. 2014 [citado em 2019 mar 8]; 38: 209-20. Disponível em: http://www.scielo.br/pdf/sdeb/v38nspe/01031104-sdeb-38-spe-0209.pdf.

Submissão: dezembro de 2016.

Aprovação: maio de 2019. 